\title{
Deep Learning Algorithms Enabling Event Detection: A Review
}

\author{
Cherifa Nakkach ${ }^{1, *}$ Amira Zrelli2,,* Tahar Ezzeddine ${ }^{3, *}$ \\ 1,2,3 National engineering School of Tunis University Tunis El Manar, Tunisia, \\ *@cherifa.nakkach@enit.utm.tn,
}

\begin{abstract}
Deep Learning has revolutionized computer vision, natural language processing, speech recognition, and information retrieval. However, as deep learning models developed, their parameter count, latency, and resource requirements rose. As a result, a model's footprint, as well as quality, should always be addressed. Academics and industry have identified neural network-based deep learning as a possible research subject. Deep learning algorithms have had tremendous results. This paper will review neural networks' deep learning methods for auditory event detection. For this reason, this paper aims to examine both highly and weakly labelled acoustic event detection systems based on deep learning. This article also discusses how deep learning might help detect events and the challenges in upcoming real-world scenarios. We briefly define the issue of model efficiency in deep learning, then cover the foundational work in the five core areas of model efficiency (modelling approaches, infrastructure, and hardware).
\end{abstract}

Keywords: Artificial intelligence, Event detection, Machine learning, Deep learning, Neural networks.

\section{INTRODUCTION}

Due to the breadth of its applications and recent technological developments, object discovery has gained increasing attention in recent years. This topic is currently the subject of substantive research in academia and real-world applications, including security surveillance, autonomous driving, transit monitoring, drone scene analysis, and robotic vision. Among the many variables and efforts that have contributed to the rapid development of object identification systems, significant contributions must be made by developing deep convolutional neural networks and the computational power of GPUs [1]. Currently, deep learning models are widely used in computer vision, including the detection of general and field-specific objects. Most modern object detectors use deep learning networks as the backbone and detection network to extract, classify, and localize properties from input images (or videos) [2]. One of the most amazing machine learning approaches now available, and deep learning has seen significant success in various applications, including image analysis, audio recognition, and text comprehension [3]. It uses supervised and unsupervised learning methodologies to learn multi-level representations and features for classification and pattern recognition tasks in hierarchical systems. Sensor networks and communication technology have recently advanced, allowing for the collection of massive amounts of data. While big data has a lot of potential in fields like e-commerce, industrial control, and intelligent medical, it also has a lot of challenges in data mining and information processing because of its high volume, variety, velocity, and veracity. During the last few years, deep learning has played a significant role in developing big data analytic solutions [4]. Deep learning is critical for big data solutions because it extracts valuable knowledge from complicated systems [5]. Deep learning has been one of the most active research topics in machine learning since its inception in 2006 [6]. Indeed, deep learning began in the 1940s. However, typical multi-layer neural network training approaches always generate an ideal local solution or cannot guarantee convergence. As a result, despite their more outstanding performance in learning and representing features, multilayer neural networks have not received broad acceptance. In [7], the authors introduced a two-stage strategy for active deep learning training, reviving deep learning for the first time. Additionally, the rise in processing power and data volume contributes to the increased interest in deep learning. As the era of big data approaches, a significant number of samples will be available for training the parameters of deep learning models. Meanwhile, training a deep learning model at scale requires high-performance computer devices. 
Consider Raina et cetera.'s network of deep beliefs. It's easy to get started with over 25 million free tutors and millions of training data. When a GPU-based framework is used, the training time for such a model is decreased from several weeks to around one day. In [8], authors mentioned that Deep learning models often acquire hierarchical qualities and huge data representations by uncontrolled pre-training and a supervised fine-tuning strategy in deep structures of classification and recognition tasks. In [9], authors mentioned that Deep learning has excelled in various applications, including computer vision, natural language processing and reading ability. Deep learning has made tremendous progress in learning the features of big data in recent years [10]. Compared to shallow machine learning approaches such as Supported Vector Machines and Naive Bayes, deep learning models may combine lowlevel inputs of vast amounts of data with high diversity and fidelity to extract high-level features and develop hierarchical representations. In [11], the authors mentioned that existing domain-specific image object detectors are divided into two types: two-stage sensors and Faster R-CNN. A single-stage detector is the other option. Achieve high object localisation and recognition accuracy using two-stage detectors while using one-stage sensors. In [12], the authors mentioned that regional pooling can split two-stage detectors into two stages. The initial step, R-CNN, is referred to as RPN or Region Proposal Network. The second stage collects features from each candidate box using RoI Pooling for classification and bounding-box regression.

We discuss and assess object detection using deep learning. Due to the rapid progress of computer vision research, the Deep Learning-Based Object Detection approach may be out of date. Our work discusses new innovative solutions but skips over the fundamentals necessary for readers to comprehend deep learning detection. Additionally, unlike past studies on object detection, this one examines deep learning-based approaches to object identification, as well as contemporary detection solutions and research trends.

\section{ARTIFICIAL INTELLIGENCE AND DEEP LEARNING}

Recent advancements in computer science shown that artificial intelligence was critical to reaching and surpassing numerous sectors [13]. To simplify the relationship between artificial intelligence, machine learning, deep learning, and neural networks, consider the example of Russian nesting dolls (figure1).

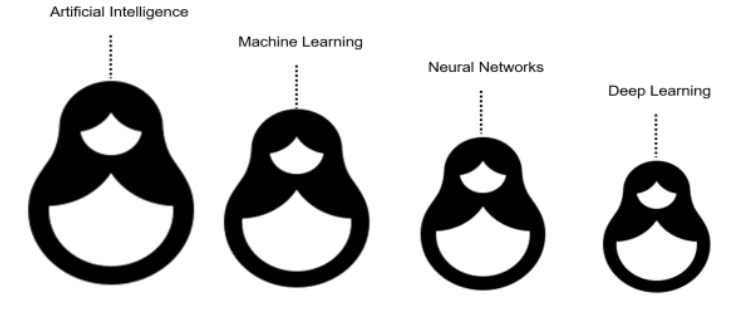

Figure 1. Artificial intelligence, machine learning, deep learning and neural network.

Machine learning (ML) is an effective way to achieve the goal of artificial intelligence. Many machine learning techniques, such as deep learning, have been created to train a machine to make classifications and predictions. It uses convolutional neural networks (ANNs).

Machine learning (ML) is a cost-effective way to achieve artificial intelligence. Several machine learning approaches, including deep learning, have been developed to teach the machine to create classifications and predictions. Neural networks (ANNs) are used.

Artificial neural networks (ANNs) or neural networks are algorithms modelled after the architecture of biological neural networks. It's a set of algorithms that try to determine the fundamental correlations in a data set using a process that simulates how the human brain works [13].

Three layers make up a neural network:

The input layer contains the raw data of the neural network.

It is the middle layer between the input and output layers. It is the site of accounts. The output layer is responsible for providing results based on the raw data. The architecture of the neural network is illustrated in Figure 2 .

Deep neural networks (ANN) or neural networks are algorithms modelled after the architecture of biological neural networks. It's a set of algorithms that try to determine the fundamental correlations in data collection using a process that simulates how the human brain works [14]. Three layers of a neural network:

- The input layer contains the raw data of the neural network.

- It is the middle layer between the input and output layers.

- It is the site of accounts.

The output layer is responsible for providing results based on the raw data. The architecture of the neural network is illustrated in Figure 2. 


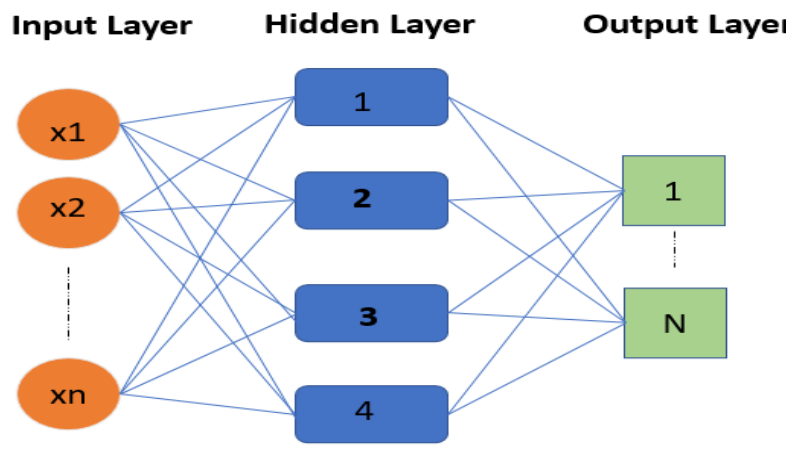

Figure 2 Architecture of a Neural Network

\subsection{Deep Learning Models}

\subsubsection{Convolution Neural Network}

In [15], the authors mentioned that the convolutional Neural Networks (CNNs) are deep learning algorithms becoming increasingly popular. It enables AI systems to automatically recognize how characteristics are extracted from inputs to perform a task, such as image classification, face authentication, and semantic segmentation of images. Figure 3 shows the construction of a convolutional neural network, and the CNN algorithm is illustrated in Figure 4.

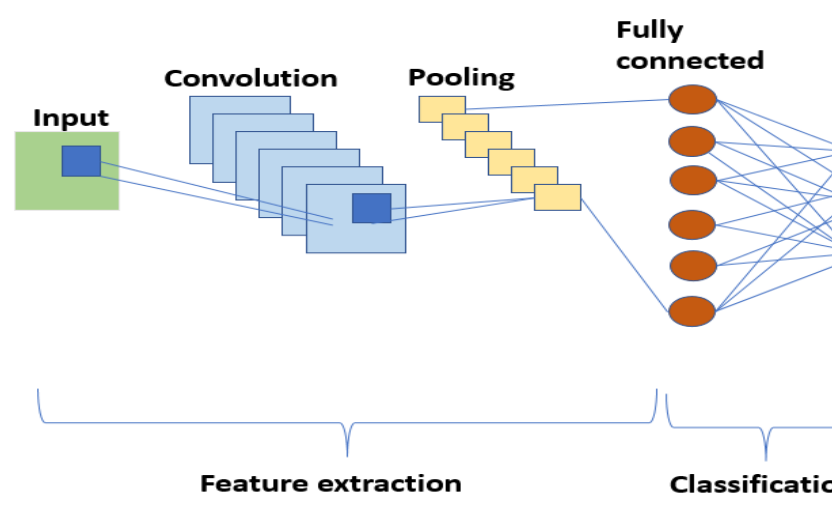

Figure 3. Structure of a Convolutional Neural Network

\begin{tabular}{l}
\hline Algorithm Of CNN \\
\hline Input Image \\
Step 1 (A) -- Convolution Operation \\
Step 1 (B) -- The Rectified Linear Unit (RELU) \\
Step 2 -- Max Pooling \\
Step 3 -- Flattening \\
Step 4 -- Full Connection \\
End \\
\hline
\end{tabular}

Figure 4. Algorithm of $\mathrm{CNN}$

\subsubsection{Recurrent Neural Network}

Recurrent neural networks (RNNs) have been created to model the time series problem. The RNN input includes the current and previous samples. Each neuron in an RNN has a memory that stores information from the neuron's previous computation. RNNs are a type of neural network that is used in natural language processing [16]. The architecture of the recurrent neural network is illustrated in Figure 5.

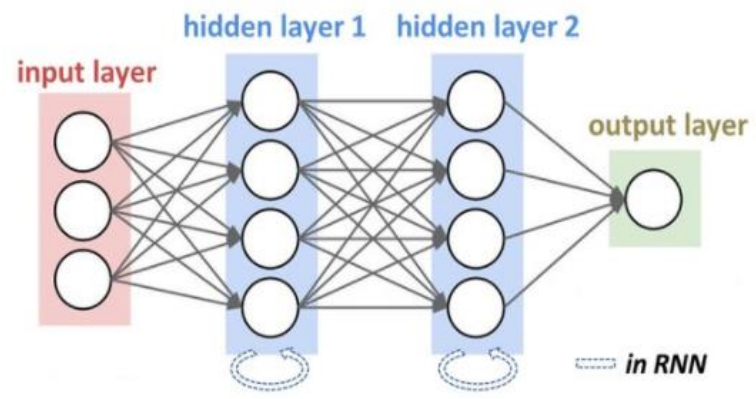

Figure 5 Structure of a Recurrent Neural Network

\subsubsection{Generative Adversarial Network}

Two components make up a Generative Adversarial Network (GAN): The generator generates reliable data that serve as passive training for the discriminator. Discriminator: It distinguishes between fictional and credible facts generated by the generator. Figure 6 illustrates the structure of the generative adversarial network [17].

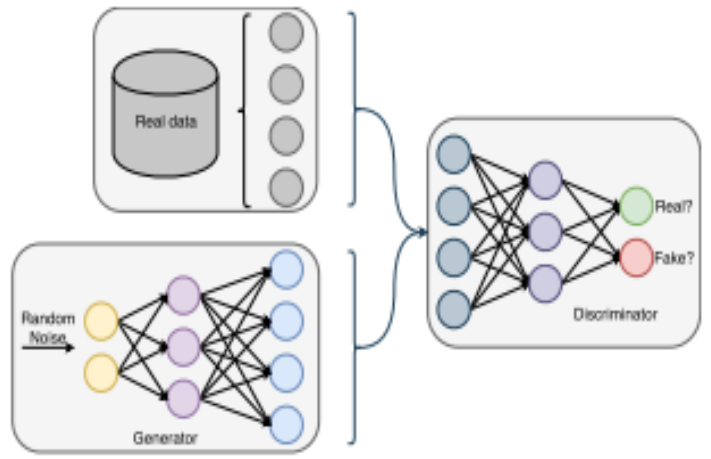

Figure 6 Structure of a Generative Adversarial Network

\subsubsection{Deep Reinforcement Learning}

DRL is the relationship between DNNs and reinforcement learning. As shown in Figure 7, it is used to solve situations in which a worker interacts with his environment in discrete time steps [18]. 


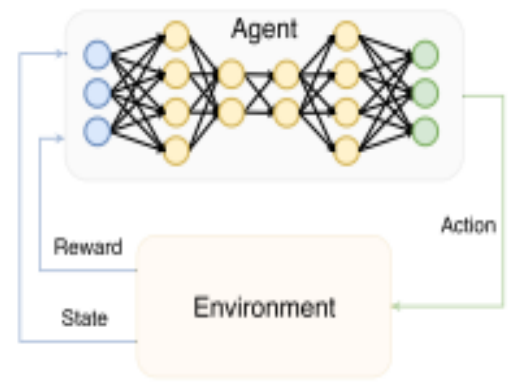

Figure 7 Deep Reinforcement Learning

\section{ALGORITHMS FOR EVENT DETECTION}

\subsection{Event detection}

An 'event' is rarely defined. There are three distinct types of "events". The first meaning may be something that happens at a specific time and place' [19]. However, it omits information about the nature and length of the event. The second relates to a particular circumstance involving humans, which includes humans in scenarios. It is widely used in computer science and applied disciplines [20], wearable sensors, health management, and business intelligence, as well as in crises, crime, media and communications, and social sciences [21]. The final definition refers to a 'change in position during the experimental evaluation [22]. Events in terms of space and time require more accurate detection and monitoring in terms of content, location, and time to help support the decision [23]. Event detection activities require various degrees of timing or performance in real-time, ranging from seconds to minutes to days. The five categories of event extraction approaches are rule-based, statistical, probabilistic, image processing, deep learning, and simulation methods [24]. We will introduce several algorithms in the next part, focusing on the deep learning method.

\subsection{Algorithms for Event Detection}

In Table 1, we summarize the accuracy of several event detection methods used in deep learning:
Table 1. Algorithms For Event Detection

\begin{tabular}{|l|l|l|l|l|}
\hline Event & Algorithm & Accuracy & Réferen \\
ce
\end{tabular}




\subsection{Discussions}

Artificial intelligence has come to prominence due to big data and intelligent algorithms. Artificial intelligence aims to develop intelligent computers capable of performing functions traditionally performed by humans. As a result, artificial intelligence has been classified as an interdisciplinary science. However, technological and educational advancements have led to near-perfect graphics in almost every sector. The robot's mind processes the input. DL and Natural Language Decoding are critical to anything from strategy game machines to autonomous vehicles. By storing vast amounts of data and creating data patterns. However, creating AI concepts and vocabulary is a challenge. Apart from the preset goals, AI is concerned with data-based (knowledge) decisions. AI has made great strides over time. On the other hand, our survey piece looks at the following developments in AI: (+) AI improves existing products. (+) Artificial Intelligence Doubles Down on Data Collection The Deep Neural Network is incredibly accurate. (+) AI discovers new information by using deep neural networks with multiple hidden layers. (+) Through revolutionary learning approaches, AI adapts data to programming. (+) AI efficiently and accurately automates repetitive learning and monitoring processes through data.

\section{CONCLUSION AND FUTURE WORK}

Without the need for any coding, machine learning can be used to improve the accuracy of programs. Using historical data, computer algorithms display the latest patterns. Machine learning enables programs to understand and learn without directly producing it. Aggregate data and data classification are just a few of the applications. Machine learning approaches are categorized into four groups based on the available data and intended learning activities.

O Supervised Artificial Intelligence (SML)

o Study under semi-supervised (SSL)

o Unsupervised Machine Learning (UML)

To summarize, deep learning technology is used in various academic fields, including speech recognition, image processing, computer vision, graphics, and medicine. This technology has become an essential tool for discovering events in the modern era. This preliminary study provides an overview of several event detection algorithms and their associated accuracy. We are now investigating several scenarios and collecting data to extend this work and apply it to real-world situations-

\section{ACKNOWLEDGMENTS}

This study is supported by the Ministry of Higher Education and Scientific Research of Tunisia. It's included in the Tunisian-South African cooperation scientific research project.

\section{REFERENCES}

[1] Korean Residential Buildings. Sustainability 2020;12(14):5573. doi:https://doi.org/10.3390/su12145573.

[2] Kusakana K. Optimal Peer-to-Peer energy sharing between prosumers usinghydrokinetic, diesel generator and pumped hydro storage. J Storage Mater2019;26. doi:https://doi.org/10.1016/j.est.2019.101048

[3] [149] Abdel-Nasser M, Mahmoud K, Kashef H. “A Novel Smart Grid State EstimationMethod Based on Neural Networks". International Journal of InteractiveMultimedia and Artificial Intelligence 2018;5(1):92. doi:https://doi.org/10.9781/ijimai.2018.01.004

[4] [150] Nawaz N. Artificial Intelligence Is Transforming Recruitment Effectiveness inCMMI Level Companies. SSRN Electronic Journal 2019. doi:https://doi.org/10.2139/ssrn.3521928

[5] . Rädle S, Mast J, Gerlach J, Bringmann O. Computational intelligence basedoptimization of hierarchical virtual power plants. Energy Syst 2020. doi:https://doi.org/10.1007/s12667-020-00382-z.

[6] Beed R, Roy A, Sarkar S, Bhattacharya D. A hybrid multi-objective tour routeoptimization algorithm based on particle swarm optimization and artificialbee colony optimization. Comput Intell 2020 . doi:https://doi.org/10.1111/coin.12276.

[7] Espe E, Potdar V, Chang E. Prosumer Communities and Relationships in SmartGrids: A Literature Review, Evolution and Future Directions. Energies2018;11(10):2528. doi: https://doi.org/10.3390/en11102528.

[8] [D. Svozil, V. Kvasnicka, and J. Pospichal. Introduction to multi-layer feed-forward neural networks. Chemometrics Intell. Lab. Syst., vol. 39, no. 1, pp. 43-[62, 1997.

[9] Allan, J., J. G. Carbonell, G. Doddington, J. Yamron, and Y. Yang. Topic Detection and Tracking Pilot Study. In proceedings of DARPA Broadcast News Transcription and Understanding Workshop, 1998.

[10] Guillem, F., and M. Mograss. Gender Differences in Memory Processing: Evidence from Event-Related Potentials to Faces." Brain and Cognition. 2005.

[11] Metzler, D., C. Cai, and E. Hovy. Structured Event Retrieval over Microblog Archives. In proceedings of the 2012 conference of the North American 
Chapter of the Association for Computational Linguistics: Human Language Technologies, Montréal, Canada, June 3-8,2012.

[12] Chen, Z., H. Ji, and R. Haralick. A Pairwise Event Coreference Model, Feature Impact and Evaluation for Event Coreference Resolution. In proceedings of the workshop on Events in Emerging Text Types, Borovets, Bulgaria, September 14-16 2009.

[13] Elkhlifi, A., and R. Faiz. French-written EventExtraction Based on Contextual Exploration.' In Twenty-Third. In Twenty-Third International Florida Artificial Intelligence Research Society Conference, Daytona Beach, Florida, USA, May 19-21. 2010.

[14] Chen, Y., L. Xu, K. Liu, D. Zeng, and J. Zhao. Event Extraction Via Dynamic Multi-Pooling Convolutional Neural Networks. In proceedings of the 53rd Annual Meeting of the Association for Computational Linguistics and the 7 th International Joint Conference on Natural Language Processing, Beijing, China, July 26-31.2015.

[15] McMinn, A. J., Y. Moshfeghi, and J. M. Jose. Building a Large-Scale Corpus for Evaluating Event Detection on Twitter.". In Proceedings of the 22nd ACM international conference on Information \& Knowledge Management,San Francisco, California, USA, October 27-November 012013.

[16]Zhou, X., and L. Chen. Event Detection over Twitter Social Media Streams. The VLDB Journal-The International Journal on Very Large Data Bases, 2014.

[17] Kopetz, H. Event-triggered Versus Time-Triggered Real-Time Systems. In Operating Systems of the 90s and Beyond, Dagstuhl Castle, Germany, July 8-12.1991.

[18] Manzhu Yu, Myra Bambacus, Guido Cervone, Keith Clarke, Daniel Duffy, Qunying Huang, Jing Li, Wenwen Li, Zhenlong Li, Qian Liu, Bernd Resch, Jingchao Yang \& Chaowei Yang (2020): Spatiotemporal event detection: a review, International Journal of Digital Earth, DOI: 10.1080/17538947.2020.1738569

[19]Eye-Movement Event Detection Meets Machine Learning R. Zemblys, 2016

[20] Acoustic Event Detection Using Machine Learning: Identifying Train Events Shannon McKenna David McLaren.

[21]Feichtl B. et al. (2020) Event Detection and Localization Using Machine Learning on a Staircase. In: Pakzad S. (eds) Dynamics of Civil Structures, Volume 2. Conference Proceedings of the Society for Experimental Mechanics Series. Springer, Cham. https://doi.org/10.1007/978-3030-12115-0_30

[22] Can Machine Learning Be Used to Recognize and Diagnose Coughs? Charles Bales, Muhammad Nabeel, Charles N. John, Usama Masood, Haneya N. Qureshi, Hasan Farooq, Iryna Posokhova, Ali Imran,2020
[23] A Deep Learning Architecture to Detect Events in EEG Signals During Sleep, Stanislas Chambon; Valentin Thorey; Pierrick J. Arnal; Emmanuel Mignot; Alexandre Gramfort,2018

[24] Prediction of Future Terrorist Activities Using Deep Neural Networks M. Irfan Uddin, Nazir Zada,2Furqan Aziz, Yousaf Saeed, Asim Zeb,4Syed Atif Ali Shah, Mahmoud Ahmad AlKhasawneh, and Marwan Mahmoud7,2020

[25] Terrorist Video Detection System Based on Faster R-CNN and LightGBM, Chao Yi, Shunxiang Wu,Bin Xi,Daodong Ming,Yisong Zhang, Zhenwen Zhou,2020

[26] Machine learning algorithms for event detection D. Margineantu, Weng-Keen Wong, D. Dash ,2010

[27] A. Krizhevsky, I. Sutskever, and G. E. Hinton. Imagenet classification with deep convolutional neural networks. in Proc. NIPS: s.n., 2012.

[28] A novel process monitoring approach based on Feature Points Distance Dynamic Autoencoder FeifanChenga, JinsongZhaoa , Computer Aided Chemical Engineering,Volume 46, Pages 757-762, 2019.

[29] I. Goodfellow et. al., Generative adversarial nets, in Proc. Adv. Neural Inf. Process. Syst. 2014.

[30] V. Mnih et. al., Human-level control through deep reinforcement learning," Nature, vol. 518, no. 7540, p. 529.2015. 